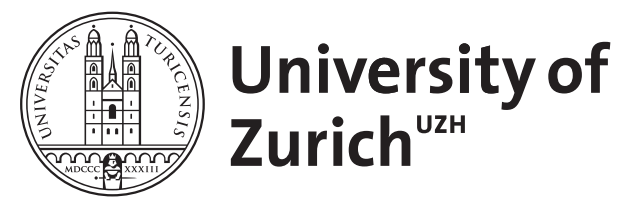

\title{
MDC1: The art of keeping things in focus
}

\author{
Jungmichel, S ; Stucki, M
}

\begin{abstract}
The chromatin structure is important for recognition and repair of DNA damage. Many DNA damage response proteins accumulate in large chromatin domains flanking sites of DNA double-strand breaks. The assembly of these structures-usually termed DNA damage foci-is primarily regulated by MDC1, a large nuclear mediator/adaptor protein that is composed of several distinct structural and functional domains. Here, we are summarizing the latest discoveries about the mechanisms by which MDC1 mediates DNA damage foci formation, and we are reviewing the considerable efforts taken to understand the functional implication of these structures.
\end{abstract}

DOI: https://doi.org/10.1007/s00412-010-0266-9

Posted at the Zurich Open Repository and Archive, University of Zurich ZORA URL: https://doi.org/10.5167/uzh-44826

Journal Article

Published Version

Originally published at:

Jungmichel, S; Stucki, M (2010). MDC1: The art of keeping things in focus. Chromosoma, 119(4):337349.

DOI: https://doi.org/10.1007/s00412-010-0266-9 


\title{
MDC1: The art of keeping things in focus
}

\author{
Stephanie Jungmichel • Manuel Stucki
}

Received: 6 January 2010/Revised: 5 February 2010 /Accepted: 14 February 2010/Published online: 12 March 2010

(C) Springer-Verlag 2010

\begin{abstract}
The chromatin structure is important for recognition and repair of DNA damage. Many DNA damage response proteins accumulate in large chromatin domains flanking sites of DNA double-strand breaks. The assembly of these structures - usually termed DNA damage foci-is primarily regulated by MDC1, a large nuclear mediator/ adaptor protein that is composed of several distinct structural and functional domains. Here, we are summarizing the latest discoveries about the mechanisms by which MDC1 mediates DNA damage foci formation, and we are reviewing the considerable efforts taken to understand the functional implication of these structures.
\end{abstract}

\section{Introduction}

The DNA, the genetic material of our cells, is constantly exposed to DNA-damaging agents such as the sun's radiation or free oxygen radicals that arise as a consequence of natural cellular metabolism. Alternatively, cells may become transiently exposed to external sources of DNA damage such as cigarette smoke or various toxic chemical compounds. DNA double-strand breaks (DSBs), which are a particularly deleterious form of DNA damage, are formed upon treatment of the cells with ionizing radiation (IR) or chemical clastogens. They are also formed in a programmed manner during meiosis and development of the immune

Communicated by E. Nigg

S. Jungmichel $\cdot$ M. Stucki $(\triangle)$

Institute of Veterinary Biochemistry and Molecular Biology,

University of Zürich,

Winterthurerstrasse 190,

8057 Zürich, Switzerland

e-mail: m.stucki@vetbio.uzh.ch system. Since DSBs are highly toxic lesions that can lead to genomic rearrangements if they are not efficiently and accurately repaired, it is not surprising that cells have evolved highly sophisticated mechanisms to counteract those threats. Based on a seminal Nature review article by Zhou and Elledge in 2000, these mechanisms are often referred to as the DNA damage response (DDR; Zhou and Elledge 2000).

The DDR is a conglomerate of signaling transduction pathways consisting of sensors, transducers, and effectors. Although it is often delineated as a linear pathway, it is more accurately described as a network of interacting pathways that together execute the cellular response. Components of the DDR in mammalian cells include the phospho-inositide-like kinases (PIKKs) ATM, ATR, and DNA-PKcs, the transducer kinases CHK1 and CHK2, and the various effector proteins that are either targeted directly by the PIKKs or by one or both of the transducer kinases. Additional proteins have emerged as essential components of the DDR eukaryotes. For example, there is a group of proteins that contain phosphorylation-specific protein-protein interaction modules such as BRCA1 carboxy-terminal (BRCT) domains and/or forkhead-associated (FHA) domains. This protein family is usually referred to as mediators/adaptors of the DDR because most of these proteins lack enzymatic activity but may predominantly act as "molecular matchmakers" through their ability to mediate the efficient interaction of proteins that would otherwise not bind to each other.

A major hallmark of the mammalian DDR is the rapid deploying of a host of proteins to the sites of DNA damage within the nuclei of affected cells. Some of these proteins engage in the repair of the lesions, while others trigger kinase-dependent signaling cascades that induce events not only confined to the region where the damage has occurred, 
but involving the entire cellular system. Some of the DDR factors that are rapidly recruited to sites of DNA damage have intrinsic affinity to aberrant DNA structures. For example, the MRE11/RAD50/NBS1 (MRN) complex, a conserved DDR sensor and repair factor, has been shown to bind preferentially to free DNA ends that arise at the sites of DSBs (de Jager et al. 2001). Other factors do not just bind to the DNA lesions per se, but accumulate in large nuclear aggregates surrounding the lesion site. These structures, which can be visualized by standard immunofluorescence microscopy, are usually referred to as nuclear foci, DNA damage foci, or ionizing radiation-induced foci (IRIF). The key regulators of IRIF formation are histone proteins that form the core of the nucleosome, the organizational unit of eukaryotic genomes. In mammalian cells, the histone $\mathrm{H} 2 \mathrm{~A}$ variant $\mathrm{H} 2 \mathrm{AX}$, a component of the nucleosome core structure that comprises $10-15 \%$ of total cellular $\mathrm{H} 2 \mathrm{~A}$ in higher organisms, is rapidly phosphorylated by PIKKs on a conserved Ser residue at its C-terminus in chromatin regions bearing DSBs. Phosphorylation of the $\mathrm{H} 2 \mathrm{AX}$ C-terminus (termed $\gamma \mathrm{H} 2 \mathrm{AX}$ ) "spreads" over large chromatin domains but is strictly confined to the damaged chromosome and does not involve neighboring chromosomes that are not affected by DNA damage (Rogakou et al. 1999). We are henceforth referring to these $\gamma \mathrm{H} 2 \mathrm{AX}$ modified chromatin regions as $\gamma \mathrm{H} 2 \mathrm{AX}$ chromatin domains. The phosphorylated $\mathrm{H} 2 \mathrm{AX} \mathrm{C}$-terminus serves as an epigenetic chromatin mark that flags regions in the genome that contain DNA breaks. Mediator of the DNA damage checkpoint 1 (MDC1; sometimes also referred to as NFBD1), a large protein that belongs to the mediator/ adaptor group of DDR factors, specifically binds to the phosphorylated H2AX C-terminus and appears to be the predominant $\gamma \mathrm{H} 2 \mathrm{AX}$ recognition factor in mammalian cells. MDC1 knockout mice display a very similar phenotype as $\mathrm{H} 2 \mathrm{AX}$ knockout mice, thus corroborating the close functional relationship between these two DDR factors (Table 1). Moreover, MDC1 is an emerging tumor suppressor because loss of MDC1 is associated with increased tumor frequency in mice (Minter-Dykhouse et al. 2008), and reduction or lack of MDC1 is observed in a significant proportion of carcinomas (Bartkova et al. 2007). MDC1 is composed of several distinct domains and regions. Each of these domains or regions seems to be tailored to recognize one or several specific protein interaction partners that are recruited to the damaged chromatin regions.

In the past 5 years, we have seen much progress in the exploration of the molecular mechanisms by which MDC1 recognizes the $\gamma \mathrm{H} 2 \mathrm{AX}$ chromatin mark and mediates the interaction of several DDR factors with damaged chromatin regions. Phosphorylation-dependent protein-protein interactions that are dependent upon FHA and BRCT domains appear to be the central theme of these processes. In this article, we will review these latest findings. For the sake of clarity, we decided to follow a structural delineation rather than a chronological account by starting from the MDC1 Cterminus and subsequently working our way up to the $\mathrm{N}$ terminus. We will briefly describe each of MDC1's structural and functional domains and regions and specify the proteins that are binding to these domains and regions. Moreover, we will summarize the functional implications of each of these domains and regions, as far as they are known. Finally, we will point out where our current knowledge about MDC1 is still incomplete and we will also highlight some of the major discrepancies in the literature.

\section{The C-terminal tandem BRCT domain: assembly and maintenance of the $\gamma \mathrm{H} 2 \mathrm{AX}$ chromatin domain, regulation of mitotic progression, and control of the decatenation checkpoint}

The tandem BRCT domain of MDC1 is located at the very C-terminus of the protein, between amino acid 1891 and 2082 (Fig. 1). X-ray structural analysis revealed that this region of MDC1 retains the typical tandem BRCT fold in which each BRCT repeat adopts a compact $\alpha / \beta$ fold and is connected by a linker region to form an extended structure about $70 \AA$ long and $35 \AA$ in diameter (Lee et al. 2005; Stucki et al. 2005). The first indication that the MDC1 tandem BRCT domain was involved in the regulation of the assembly of the $\gamma \mathrm{H} 2 \mathrm{AX}$ chromatin domain came from the observation that a green fluorescent protein (GFP) tagged version of the protein lacking the $\mathrm{C}$-terminal $\mathrm{BRCT}$ region did not accumulate in IRIF in response to IR treatment. Moreover, the same study showed that overexpression of the BRCT region abrogated MDC1 and $\gamma \mathrm{H} 2 \mathrm{AX}$ IRIF (Shang et al. 2003).

Since BRCT repeats can act as phosphopeptide-binding domains, oriented phosphopeptide library screening was used to define the optimal phosphopeptide binding motif for the MDC1 BRCT tandem domain (Rodriguez et al. 2003; Stucki et al. 2005). These screens revealed that the MDC1 tandem BRCT domain bound selectively to peptides containing a phosphorylated Ser residue, and furthermore, selected for Glu at the +2 position and Tyr at the +3 position after the phosphorylated serine. These binding preferences closely match the sequence of the phosphorylated $\mathrm{H} 2 \mathrm{AX} \mathrm{C}$-terminus (pS-Q-E-Y-COOH), suggesting that $\gamma \mathrm{H} 2 \mathrm{AX}$ is one of the binding partners of the MDC1 tandem BRCT domain. Indeed, biochemical, X-ray structural, and cell biological approaches clearly demonstrated that MDC1 directly interacts with $\gamma \mathrm{H} 2 \mathrm{AX}$ via its Cterminal BRCT region (Lee et al. 2005; Lou et al. 2006; 
Table 1 Phenotypes associated with MDC1 loss. MDC1 loss induces a multitude of phenotypes both on the cellular level and also on the level of the whole organism

\begin{tabular}{lll}
\hline KO organism (mouse) & KO cellular (mouse) & siRNA cellular (human) \\
\hline Small size & Radiosensitivity & Radiosensitivity \\
Male infertility & Intra-S phase checkpoint & Intra-S phase checkpoint \\
Radiosensitivity & G2/M checkpoint & G2/M checkpoint \\
Tumor-prone & IRIF & IRIF \\
& Phosphorylation (CHK1, CHK2, ATM) & Phosphorylation (CHK1, CHK2, ATM, SMC1) \\
& DSB repair by HR & DSB repair by HR \\
& NHEJ of dysfunctional telomeres & Random plasmid integration \\
& Chromosomal instability & Apoptosis \\
& Reduced proliferation & Slow mitosis \\
& Class switch recombination &
\end{tabular}

In this table we summarized the most important phenotypes associated with MDC1 loss

Stucki et al. 2005) (reviewed in Stucki and Jackson 2006). The structural data revealed that the phospho-peptide binding cleft of the MDC1 tandem BRCT domain is exquisitely tailored to recognize the $\gamma \mathrm{H} 2 \mathrm{AX}$ motif (Lee et al. 2005; Stucki et al. 2005). Point mutations in the phosphopeptide binding cleft of the MDC1 BRCT tandem domain, as well as in the H2AX C-terminus, abrogated the $\mathrm{MDC} 1-\gamma \mathrm{H} 2 \mathrm{AX}$ interaction in vitro; disrupted IRIF formation by several DDR factors including MDC1 itself, the MRN complex, phosphorylated ATM, 53BP1, and BRCA1; and rendered cells radio-sensitive (Stucki and Jackson 2006). These findings strongly indicate that one of the major biological functions of the MDC1 C-terminal BRCT region is the initial recognition of the $\gamma \mathrm{H} 2 \mathrm{AX}$ chromatin mark and the mediation of the recruitment and retention of DDR factors in chromatin regions flanking DSBs.

MDC1 does not only recognize the $\gamma \mathrm{H} 2 \mathrm{AX}$ chromatin mark, but it is also involved in its regulation. It was shown that MDC1 loss or experimental disruption of the MDC1 $-\gamma \mathrm{H} 2 \mathrm{AX}$ interaction leads to reduced H2AX phosphorylation and small $\gamma \mathrm{H} 2 \mathrm{AX}$ IRIF (Lou et al. 2006; Stewart et al. 2003; Stucki et al. 2005). This indicates that MDC1 somehow positively regulates H2AX phosphorylation and that this regulation is dependent on the direct interaction between MDC1 and $\gamma \mathrm{H} 2 \mathrm{AX}$. Since $\mathrm{H} 2 \mathrm{AX}$ phosphorylation in response to DSBs does not spread in three dimensions throughout the cell nucleus, but is confined to chromatin regions flanking the break site on

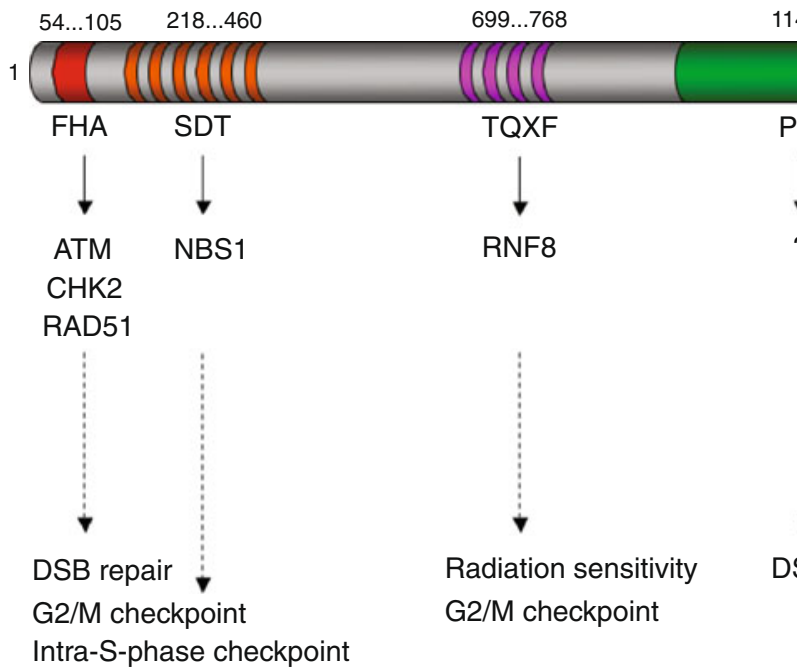

Fig. 1 Schematic representation of the domain architecture of MDC1 and its interaction partners. MDC1 is composed of several distinct domains and regions that either interact with phosphorylated proteins or are themselves phosphorylated and serve as docking sites for other

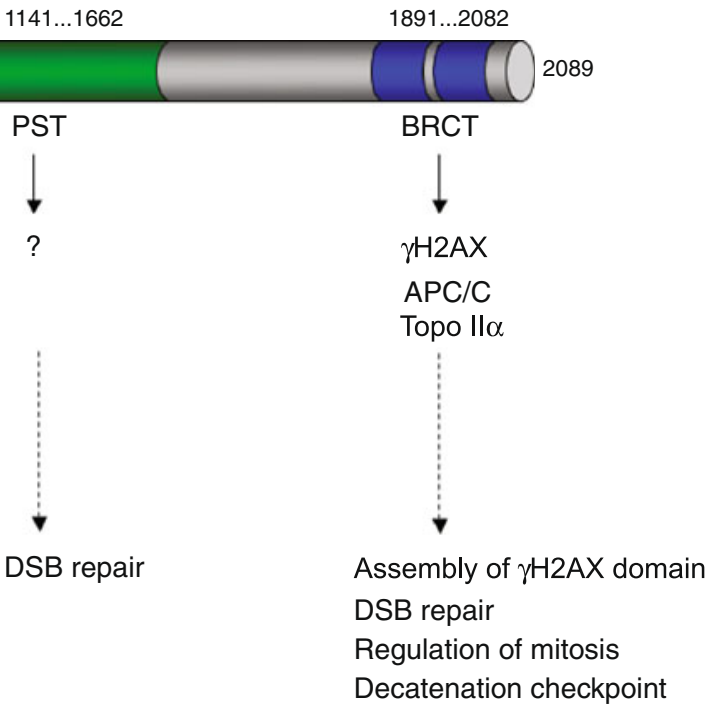

proteins that contain phospho-specific interaction modules such as FHA and BRCT domains. Each of these domains and regions appears to be functionally relevant for the DDR and/or for control of the cell cycle. See text for details 
the damaged chromosome (i.e. "spreads" only in two dimensions), it was proposed that MDC1 may be required for $\gamma \mathrm{H} 2 \mathrm{AX}$ spreading along the damaged chromatin fiber (Lou et al. 2006; Stucki and Jackson 2006). According to this model, MDC1 is recruited to $\gamma \mathrm{H} 2 \mathrm{AX}$ proximal to the lesion and either directly or indirectly mediates the recruitment and retention of activated ATM in the damaged chromatin compartment. ATM could then phosphorylate more $\mathrm{H} 2 \mathrm{AX}$ molecules that are located more distal to the initiating lesion. As a consequence, the $\gamma \mathrm{H} 2 \mathrm{AX}$ chromatin mark would spread further and further away from the initial break. However, the model of MDC1-fuelled self-reinforcing $\mathrm{H} 2 \mathrm{AX}$ phosphorylation cycles to spread the $\gamma \mathrm{H} 2 \mathrm{AX}$ chromatin mark along the chromosome axis has recently been challenged. One of the major caveats in assessing the mechanism of $\gamma \mathrm{H} 2 \mathrm{AX}$ formation was the lack of "resolution." Up until recently, researchers have relied on microscopic techniques to study the dynamic assembly of the $\gamma \mathrm{H} 2 \mathrm{AX}$ chromatin domain. While these microscopic techniques have been very useful to measure the kinetics of protein recruitment to sites of DNA damage and to assess the dynamics of the assembly and disassembly of the $\gamma \mathrm{H} 2 \mathrm{AX}$ chromatin domain, they have not been capable of delivering any information about the density of $\mathrm{H} 2 \mathrm{AX}$ phosphorylation and the distance of $\gamma \mathrm{H} 2 \mathrm{AX}$ spreading along the axis of the damaged chromosome. However, there exists a very powerful technique that can yield this kind of data: chromatin immunoprecipitation (ChIP). While ChIP has been widely and successfully used to study the assembly of protein complexes on chromatin, as well as the dynamic changes of epigenetic states (e.g., in transcriptional control), its successful application in the mammalian DDR field has long been impeded by the challenge to efficiently induce DNA breaks at specific loci in the genome. To circumvent this problem, one group studied the spreading of $\gamma \mathrm{H} 2 \mathrm{AX}$ on shortened telomeres in senescent primary cells (Meier et al. 2007). Shortened telomeres in senescent cells have been shown to trigger a DDR similar to the one that is activated by DSBs. In this study, it was shown that the $\gamma \mathrm{H} 2 \mathrm{AX}$ chromatin mark spreads up to about $570 \mathrm{~kb}$ into the subtelomeric regions. Furthermore, the spreading pattern of MDC1 was very similar to that of $\gamma \mathrm{H} 2 \mathrm{AX}$, confirming the functional and structural link between these two factors. Importantly, this study also revealed that $\gamma \mathrm{H} 2 \mathrm{AX}$ density is not uniform along the chromosome axis (Meier et al. 2007). Another study recently used primary mouse lymphocytes to study $\gamma \mathrm{H} 2 \mathrm{AX}$ density and spreading during $\mathrm{V}(\mathrm{D}) \mathrm{J}$ recombination in G1 (Savic et al. 2009). The major advantage of this experimental system is the efficiency by which the RAG1/ RAG2 (RAG) endonucleases induce DSBs between the variable $(\mathrm{V})$, diversity $(\mathrm{D})$, and joining $(\mathrm{J})$ gene segments and their flanking RAG recognition sequences. Since the genomic locations of the RAG-initiated DSBs are known, $\gamma \mathrm{H} 2 \mathrm{AX}$ density and spreading could readily be measured by ChIP at this specific locus. Surprisingly, these data revealed that MDC1 is not required for the spreading of the $\gamma \mathrm{H} 2 \mathrm{AX}$ chromatin mark but, instead, for keeping up a high $\gamma \mathrm{H} 2 \mathrm{AX}$ density proximal to the break site (Savic et al. 2009). Thus, it is possible that the previously proposed MDC1-dependent $\gamma \mathrm{H} 2 \mathrm{AX}$ self-reinforcing mechanism only applies to chromatin regions close to the lesion, while other mechanisms may regulate $\gamma \mathrm{H} 2 \mathrm{AX}$ spreading into more distal regions of the damaged chromatin fiber. It remains to be determined whether these observations only apply to RAG-initiated DSBs or if they are a general characteristic of the cellular response to DSBs.

It has been proposed that MDC1 may control H2AX phosphorylation through another mechanism that is not related to its ability to mediate the accumulation of active ATM in damaged chromatin compartments. This model suggests that MDC1 may control the dephosphorylation of $\mathrm{H} 2 \mathrm{AX}$ through its direct binding to the phospho-epitope located at C-terminus of H2AX. This interpretation was based on the observation that the purified MDC1 BRCT domains could efficiently shield $\gamma \mathrm{H} 2 \mathrm{AX}$ phosphopeptides from phosphatase activity in vitro. Moreover, overexpression of the isolated MDC1 BRCT region in mammalian cells resulted in H2AX hyperphosphorylation (Stucki et al. 2005). It was recently discovered that the phosphatases PP2A and PP4 are involved in the removal of $\gamma \mathrm{H} 2 \mathrm{AX}$ from chromatin in mammalian cells (Nakada et al. 2008). Thus, it will be interesting to see if MDC1 can limit the access of these phosphatases to $\gamma \mathrm{H} 2 \mathrm{AX}$ in vivo.

The recent discovery of yet another phosphorylation event at the H2AX C-terminus has added an additional layer of complexity to the regulation of the $\gamma \mathrm{H} 2 \mathrm{AX}$ chromatin domain. In two recent Nature articles, the Allis and Rosenfeld laboratories described the phosphorylation of Tyr142 (the very C-terminal amino acid of H2AX) by the WICH complex and its dephosphorylation by the EYA1/3 phosphatases (Cook et al. 2009; Xiao et al. 2009). As these findings and their implications for the DDR have recently been reviewed in detail elsewhere (Stucki 2009), we will just briefly summarize the main issues here: while H2AX Ser139 is targeted by DNA damage-activated PIKKs, Tyr142 is phosphorylated by the constitutive kinase WSTF, a subunit of the WICH chromatin remodeling complex. However, upon induction of DNA damage, Tyr142 becomes dephosphorylated by the EYA1/3 phosphatases. How exactly this dephosphorylation is regulated remains elusive, but Tyr142 dephosphorylation is necessary for the assembly of the $\gamma \mathrm{H} 2 \mathrm{AX}$ chromatin domain because MDC1 is not capable of efficiently binding to $\gamma \mathrm{H} 2 \mathrm{AX}$ as long as Tyr142 is phosphorylated.

But what is the physiological role of H2AX Tyr142 phosphorylation? One attractive possibility is that the 
Tyr142 phosphorylation/dephosphorylation equilibrium may determine cell fate in response to genotoxic stress. As mentioned above, Tyr142 phosphorylation attenuates MDC1 recruitment and the establishment of the $\gamma \mathrm{H} 2 \mathrm{AX}$ chromatin domain. However, it was proposed that the Tyr142 modification may promote the recruitment of the pro-apoptotic factor JNK1 to sites of DNA damage (Cook et al. 2009). Thus, if repair is possible, Tyr142 is dephosphorylated and the DDR machinery is recruited to sites of DNA damage to promote repair. However, if the damage is too severe to be repaired in time, tyrosine phosphorylation remains and apoptosis is favored through the association of JNK1 with $\gamma \mathrm{H} 2 \mathrm{AX}$. This is still a rather simplified model, and our understanding of the regulation of the $\gamma \mathrm{H} 2 \mathrm{AX}$ chromatin domain is still incomplete. Thus, more work is needed to understand this important process in detail.

Recently, two additional phosphorylation-specific interaction partners for the MDC1 BRCT domains have been discovered. $\mathrm{CDC} 27$, a subunit of the anaphase promoting complex APC/C was shown to interact directly with MDC1 via its phosphorylated C-terminal region (Coster et al. 2007). The APC/C is a multi-subunit E3 ubiquitin ligase that controls mitotic progression mainly through ubiquitindependent destruction of mitotic cyclins and other substrates in a coordinated manner. Interestingly, the sequence of the phosphorylated MDC1 binding site in $\mathrm{CDC} 27$ is located at the very $\mathrm{C}$-terminus of the protein and is very similar to the MDC1 binding site in H2AX: the phosphoacceptor Ser residue is also followed by a Glu at the +2 position and a Phe at the +3 position (pS-D-E-F-COOH). Phosphopeptide screening has indicated that the MDC1 BRCT domains strongly select for Glu at +2 , and Tyr, but not Phe, at the +3 position (Rodriguez et al. 2003; Stucki et al. 2005). Thus, it appears that CDC27 binding to MDC1 is effectively produced by the strong selection of Glu at +2 . Moreover, the relative localization of the $\mathrm{C}$-terminal carboxylate in respect to the phosphorylated Ser residue is identical in $\mathrm{CDC} 27$ and $\gamma \mathrm{H} 2 \mathrm{AX}$. The kinase that targets the CDC27 C-terminus is currently unknown, but considering that the MDC1 CDC27 interaction is increased after DNA damage (Coster et al. 2007), CDC27 phosphorylation may be somehow modulated by DNA damage. An interesting possibility would be that MDC1 is involved in the regulation of mitotic progression in response to DNA damage through its interaction with the APC/C. However, no experimental evidence for such a function has yet been produced. Instead, it was recently demonstrated that MDC1 is regulating mitotic progression independently of DNA damage. Grant Stewart and colleagues showed that MDC1deficient human cells have defects in mitotic progression at the metaphase/anaphase transition (Townsend et al. 2009). Moreover, the activity of the $\mathrm{APC} / \mathrm{C}$ ubiquitin ligase is compromised in the absence of MDC1. However, mechanistically, MDC1 appears to modulate the APC/C activity rather through its interaction with $\mathrm{CDC} 20$ (an activator protein that regulates the substrate specificity of the APC/C ubiquitin ligase) and not through its interaction with the APC/C subunit CDC27 (Townsend et al. 2009). Thus, it remains elusive as to how the phospho-dependent MDC1CDC27 interaction contributes to the control of mitotic progression.

Another phospho-specific MDC1 BRCT-interacting factor was recently discovered by Lou and colleagues: DNA topoisomerase II $\alpha$ (Topo II $\alpha$; (Luo et al. 2009)). MDC1Topo II $\alpha$ interaction seems to regulate the decatenation checkpoint, a checkpoint that controls the entanglement ("catenation") of chromosomes at the end of DNA replication. This checkpoint arrests cells in G2 and delays onset of mitosis until sister chromatids are fully separated. A phosphorylation site close to the C-terminus of Topo II $\alpha$ appears to mediate the interaction with the MDC1 Cterminal BRCT repeat. Surprisingly though, this phosphorylation site presents only limited sequence similarity to the $\mathrm{H} 2 \mathrm{AX}$ and $\mathrm{CDC} 27$ C-terminal MDC1-binding epitopes, suggesting a different mode of binding, or perhaps an indirect interaction. Quantitative phosphopeptide-binding studies and/or structural approaches will be required to resolve this issue.

\section{The PST repeat region: regulation of DSB repair and mitotic progression?}

The region in human MDC1 between amino acids 1141 and 1662 is generally referred to as the proline-serine-threonine (PST)-rich repeat (Fig. 1). In humans, this region consists of 13 consecutive imperfect repeats of 41 amino acids. The PST repeat is one of the least conserved regions in MDC1 and does not appear to exist at all in MDC1 orthologues of non-vertebrates (e.g., Drosophila (Dronamraju and Mason 2009)). However, in most vertebrate species, the PST repeat is present and conserved, even though the number of repeats varies greatly from 13 consecutive repeats in human MDC1 to seven repeats in mouse MDC1. Primary sequence analysis did not reveal any known structural and functional motifs, and sequence comparison and database searches did not retrieve significant homology to any other known protein.

To this day, little is known about the functional implication of the PST repeat region. However, it is clear that the PST repeat is neither required for MDC1 accumulation at sites of DSBs (Shang et al. 2003), nor for MDC1's function to mediate the accumulation of 53BP1, BRCA1, and the MRN complex in damaged chromatin compartments (Lou et al. 2004; Xie et al. 2007) (see also 
next sections). In an early study by the Chen laboratory, it was proposed that the PST repeat region constitutes a binding site for DNA-PK. Moreover, in this work, it was shown that deletion of the PST repeat triggers a partial nonhomologous end joining (NHEJ) defect (Lou et al. 2004). However, the mechanism by which the MDC1 PST repeat would regulate NHEJ remained elusive because MDC1 is not required for DNA-PK recruitment to sites of DSBs (Gottlieb and Jackson 1993). It was proposed that MDC1 may mediate the accumulation of DNA-PK in chromatin regions flanking DSBs in similar manner as it mediates the accumulation of the MRN complex (Lou et al. 2004). However, DNA-PK does not seem to accumulate in IRIF in response to DSBs (Bekker-Jensen et al. 2006). Thus, a functional implication of the observed interaction between the MDC1 PST region and DNA-PK remains elusive.

Interestingly, the PST repeat region does not only appear to be important for DSB repair by NHEJ, but also for homologous recombination (HR). In a genetic HR reporter system, a mutant MDC1 version lacking the PST repeat region was defective for $\mathrm{HR}$, while this mutant was still proficient in accumulating at sites of DSBs and in mediating the accumulation of other DDR proteins such as 53BP1, BRCA1 at sites of DSBs (Xie et al. 2007). It thus appears that the MDC1 PST repeat region is implicated in DSB repair by both NHEJ and HR, although the mechanism of action of this unusual repeat motif in DSB repair remains to be elucidated.

According to a recent report, the PST repeat region is also implicated in mitotic progression. As outlined in the last section, MDC1 deficient cells have defects in mitotic progression at the metaphase/anaphase transition, which is associated with an increased number of mitotic cells in a population of MDC1 deficient cells as compared to control cells that express normal amounts of MDC1 (Townsend et al. 2009). Expression of a mutant MDC1 version lacking the PST repeat region could not restore this mitotic defect while expression of wild-type MDC1 rescued the phenotype. Interestingly, there seems to be an interaction between several subunits of the $\mathrm{APC} / \mathrm{C}$ and the MDC1 PST repeat region, which may mechanistically explain the mitotic defect observed in cells expressing a MDC1 version lacking the PST repeat (Townsend et al. 2009).

\section{The TQXF cluster: Regulation of chromatin ubiquitylation}

N-terminal of the PST repeat resides a region in MDC1 that is characterized by a noticeable abundance of PIKK consensus phosphorylation Ser/Thr-Gln (S/TQ) sites. Particularly striking is a cluster of four nearby TQ motifs that are all followed by the Phe at the +3 position (located between amino acid 699-768 in human MDC1). We will henceforth refer to this region as the TQXF cluster. Early studies on human MDC1 suggested that the protein is targeted by ATM in response to IR treatment, but the exact location of the phosphorylation sites were not determined (reviewed in Stucki and Jackson 2004). However, at least a subset of the TQXF motifs in MDC1 have recently been identified to represent bona fide ATM targets in response to IR (Kolas et al. 2007; Mailand et al. 2007; Matsuoka et al. 2007). Phosphorylation of these sites mediates the recruitment of the E3 ubiquitin ligase RNF8 through direct interaction of its FHA domain with the MDC1 phosphorylated TQXF region (Huen et al. 2007; Kolas et al. 2007; Mailand et al. 2007) (see Fig. 2). FHA domains, like BRCT tandem domains, recognize amino acid sequences around a central phosphorylation site. However, while BRCT tandem domains recognize both pSer and pThr-containing sequences, FHA domains appear to only recognize pThr-containing motifs (Durocher and Jackson 2002). Intriguingly, the RNF8 FHA domain showed strong selection for Phe and Tyr at the +3 position (Huen et al. 2007). Such a selection for aromatic amino acids at the +3 position closely resembles the optimal phosphopeptide motifs recognized by the tandem BRCT domains of MDC1 and BRCA1 (reviewed in Mohammad and Yaffe 2009; see also above).

Immunofluorescence microscopy showed that deletion of these TQXF motifs in MDC1 or depletion of RNF8 led to impaired IRIF formation for conjugated ubiquitin, 53BP1 and BRCA1, whereas $\gamma \mathrm{H} 2 \mathrm{AX}, \mathrm{MDC} 1$, and NBS1 IRIF were not affected. Furthermore, 53BP1 and BRCA1 accumulation was dependent on the E3 ubiquitin ligase domain (a RING domain) of RNF8, indicating that IRIF formation of these proteins is controlled by local ubiquitylation of substrates in chromatin regions flanking DSBs. Although the identity of these substrates has currently not yet been addressed in vivo, several lines of in vitro evidence suggest that the major RNF8 substrates that mediate 53BP1 and BRCA1 accumulation at sites of DSBs may be the histone proteins $\mathrm{H} 2 \mathrm{~A}$ and $\mathrm{H} 2 \mathrm{AX}$ (Huen et al. 2007; Mailand et al. 2007).

What is the physiological role of local chromatin ubiquitylation and how exactly does this posttranslational modification influence the accumulation of DDR proteins such as 53BP1 and BRCA1 in damaged chromatin regions? Downregulation of RNF8 by small interfering RNAs (siRNAs) yields a $\mathrm{G} 2 / \mathrm{M}$ checkpoint defect and renders cells radiosensitive, implicating MDC1-dependent local chromatin ubiquitylation in DNA damage signaling and perhaps also DSB repair in mammalian cells. The mechanism, by which MDC1-dependent RNF8 recruitment ensues the chromatin ubiquitylation cascade and mediates IRIF formation by 53BP1 and BRCA 1 appears to be rather complicated and has yet to be resolved in detail. However, 
1. Initial recruitment of MRN to DSB and ATM activation

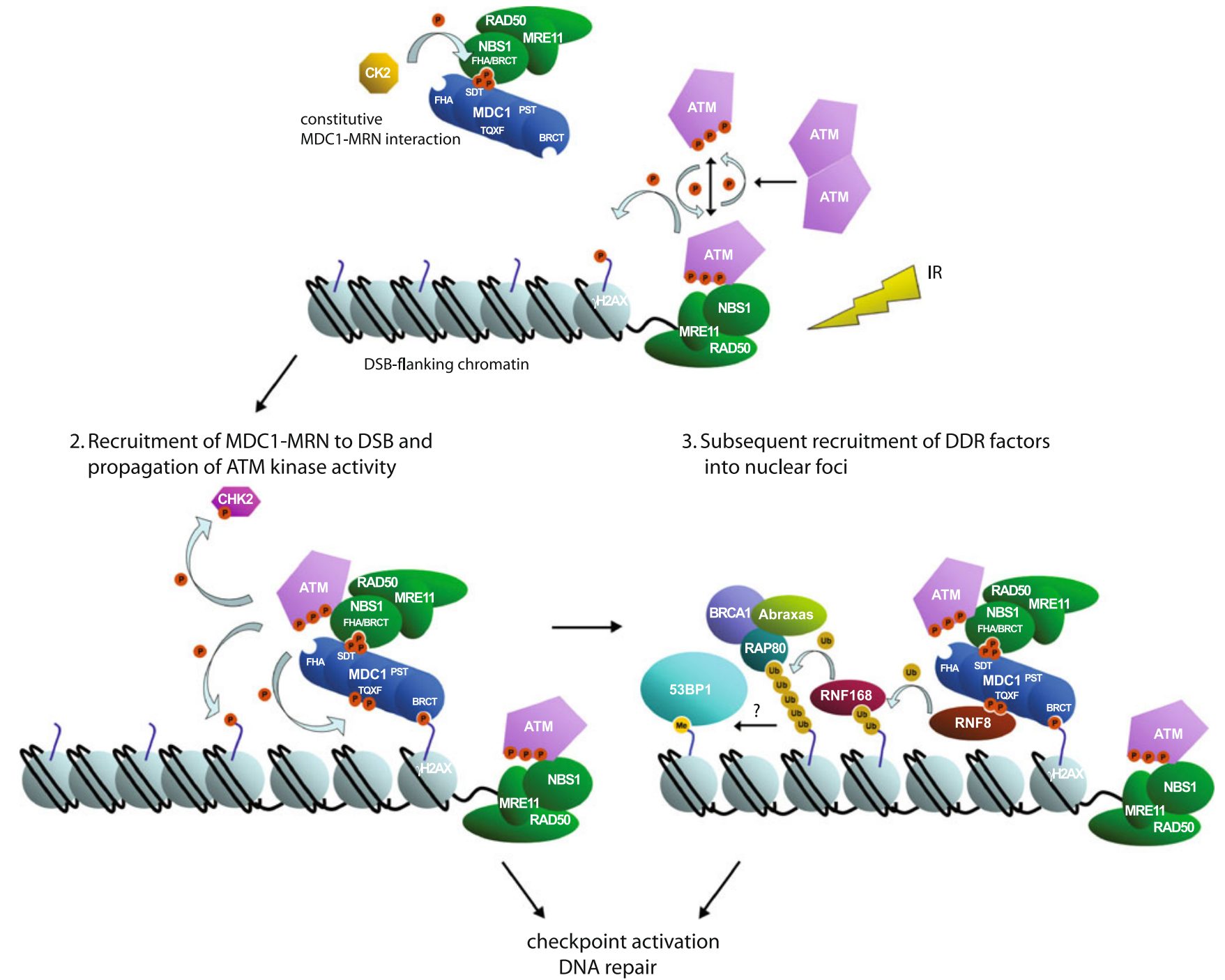

Fig. 2 Model of the assembly of the $\gamma \mathrm{H} 2 \mathrm{AX}$ chromatin domain. $1 \mathrm{In}$ response to DSB-causing genotoxic agents MRN directly binds to free DNA ends and facilitates the recruitment and activation of the ATM kinase, which is autophosphorylated and, thus, converted from an inactive dimer into active monomers. ATM phosphorylates $\mathrm{H} 2 \mathrm{AX}$ in the nearby DSB-flanking chromatin. $2 \mathrm{MDC} 1$ recognizes the $\gamma \mathrm{H} 2 \mathrm{AX}$

it is clear that at least one additional ubiquitin ligase and ubiquitin-dependent adaptor proteins are involved in these processes. As these downstream events are not directly controlled by MDC1 and have been recently reviewed in detail elsewhere (Panier and Durocher 2009; Van Attikum and Gasser 2009), we will just briefly summarize the most important findings here and outline the current model (see Fig. 2).

While it was shown that the ubiquitin ligase activity of RNF8 is required to trigger DSB-associated ubiquitylation, it is not sufficient to sustain the conjugated ubiquitin in the damaged chromatin regions. Several groups identified RNF168 as a novel chromatin-associated ubiquitin ligase chromatin mark and mediates the sustained interaction of the MRN complex with damaged chromatin through direct phosphorylationdependent interaction. 3 Phosphorylated MDC1 recruits the ubiquitin ligase RNF8 and additional DDR factors, thereby triggering a signaling cascade that is dependent on ubiquitylation of histones and results in checkpoint activation and DNA repair. See text for details

that acts in concert with UBC13 to catalyze formation of K63-linked ubiquitin conjugates and ubiquitylates family members of the $\mathrm{H} 2 \mathrm{~A}$ family in regions surrounding the DSB as efficiently as RNF8. In addition, RNF168 possesses the ability to bind ubiquitylated H2A in an RNF8dependent manner (Doil et al. 2009; Stewart et al. 2009). This suggests that, while RNF8 acts as initiating ubiquitin ligase in DSB-flanking chromatin, RNF168 activity stabilizes and/or amplifies the signal in order to reach a threshold level that mediates the recruitment of downstream factors 53BP1 and BRCA1 and thereby allows the completion of the DSB-induced chromatin response. Significantly, RNF168 is mutated in RIDDLE syndrome patients that 
suffer from immunodeficiency and radiosensitivity (Stewart et al. 2007, 2009). These phenotypes may arise mainly from defective chromatin ubiquitylation and dysfunctional 53BP1 and BRCA1 retention, a process that may be particularly important for the proper repair of programmed DSBs during the development the immune system in humans (recently reviewed in Stewart 2009).

The ubiquitylated histones likewise form a direct recognition motif for the ubiquitin-interacting motif (UIM)-containing receptor-associated protein 80 (RAP80), leading to its accumulation in IRIF with a preference for K63- and K6-linked ubiquitin polymers (Kim et al. 2007; Sobhian et al. 2007; Wang et al. 2007). RAP80 accumulates in IRIF and facilitates the recruitment of BRCA1, which is mediated by Abraxas that directly interacts with the BRCT domain of BRCA1 in a DNA damage-induced phosphodependent manner (Wang et al. 2007) (Fig. 2). Taken together, the sequential recruitment of the three ubiquitin ligases RNF8, RNF168, and BRCA1 and the resulting ubiquitylation of chromatin components (perhaps mainly H2A-type histones) imply that this type of posttranslational modification represents a crucial novel regulatory process in the response to genotoxic lesions.

While the MDC1/RNF8-dependent retention of BRCA1 can be mechanistically explained with a model that involves direct protein-protein interactions, the MDC1/ RNF8-dependent accumulation of 53BP1 is less well understood. No ubiquitin-binding domains have yet been identified in 53BP1 that could explain its recruitment to DSB-associated ubiquitylated chromatin components. Thus, MDC1 may exert its function also more indirectly, e.g., through initiation of chromatin reorganization events that would eventually increase the accessibility of proteins to newly exposed histone marks that are buried in higherorder chromatin structure. In this regard, the 53BP1 Tudor domain was shown to be required for 53BP1 accumulation at sites of DSBs (Huyen et al. 2004). These domains may bind to methylated histone marks that are masked in unperturbed chromatin, but may become locally exposed in a ubiquitin-dependent manner in response to DNA damage (Botuyan et al. 2006; Huyen et al. 2004). In any case, the vast majority of data presently available promote the idea that MDC1-mediated chromatin modifications and/or higherorder chromatin rearrangements might facilitate the accumulation and sustained retention of 53BP1 at sites of DSBs.

What is the functional implication of MDC1/RNF8mediated BRCA1 and 53BP1 accumulation at sites of DSBs? While this is still not yet resolved in detail, an interesting clue recently came from the telomere field. It has been known for quite some time that chromosomes that have lost their protective telomeric "cap" undergo chromosome end-to-end fusions in a process that is dependent on the NHEJ machinery. MDC1-deficient cells show a significant reduction in NHEJ of dysfunctional telomeres (Dimitrova and de Lange 2006). This is most likely due to the inefficient accumulation of 53BP1 at dysfunctional telomeres in the absence of MDC1. 53BP1 is thought to promote chromosome end-to-end fusions by altering the dynamic behavior of chromatin at dysfunctional telomeres (Dimitrova and de Lange 2008). However, it is not yet known if this effect is specific for NHEJ of dysfunctional telomeres or if it also applies to NHEJ in general.

\section{The SDT repeat region: constitutive interaction with the MRN complex}

Upstream of the TQXF cluster, MDC1 features a second repeat region that is characterized by conserved patches of 8-10 amino acids comprising Ser and Thr residues typically separated by an aspartate and further embedded in an acidic sequence environment. This so called SDT region (in some papers also referred to as SDTD region) interacts with the MRN complex in a phosphorylation-dependent manner (Chapman and Jackson 2008; Melander et al. 2008; Spycher et al. 2008; Wu et al. 2008) (Fig. 2). The MRN complex is an essential component of the DNA damage signaling machinery. Due to its ability to recognize free DNA ends, the MRN complex is capable of acting as a sensor of DSB. It further exhibits DNA-end processing activity, tethers DNA ends, and is required for efficient ATM kinase activation (reviewed in Williams et al. 2007). Accomplishing the aforementioned functions does not seem to require the presence of MDC1. However, similar to 53BP1 and BRCA1 (see above), accumulation and retention of MRN complex in DSB-flanking chromatin regions strictly depends on MDC1 (Bekker-Jensen et al. 2006; Goldberg et al. 2003; Lukas et al. 2004).

In human MDC1, six SDT motifs were identified, and deletion of at least five of them leads to complete abrogation of MRN IRIF formation (Melander et al. 2008; Spycher et al. 2008). Analysis of NBS1 recruitment to sites of DSBs revealed that, upon expression of an MDC1 version lacking the SDT regions, NBS1 only accumulates in micro-IRIF but is not found in the broader chromatin compartments usually covered by $\gamma \mathrm{H} 2 \mathrm{AX}$ and MDC1 (Chapman and Jackson 2008). This indicates that the MRN complex is recruited to DSBs in a MDC1independent manner, but its sustained interaction with the DSB-flanking chromatin requires MDC1.

Interestingly, MDC1 and MRN exist in a complex even in undamaged cells. This interaction is dependent on the activity of the acidophilic caseine kinase 2 (CK2) for which the SDT motifs form consensus phosphorylation sites (Spycher et al. 2008; Wu et al. 2008). Both Ser and Thr residues in each SDT motif are phosphorylated by CK2 in 
vivo, and only doubly phosphorylated pSDpT motifs are capable of mediating the interaction with NBS1 (Melander et al. 2008; Spycher et al. 2008).

Two recent X-ray structural studies revealed that NBS1 contains a compact module at its $\mathrm{N}$-terminus formed by an FHA and a tandem BRCT domain (Lloyd et al. 2009; Williams et al. 2009). Phosphopeptide-binding studies revealed that both the FHA domain of NBS1 and its BRCT domains preferentially bind to SDT-like motifs that are phosphorylated on both Ser and Thr residues (Lloyd et al. 2009). These motifs are found in MDC1 (see above), and also in fission yeast Ctp1 (the yeast orthologue of mammalian CtIP that is involved in DSB resection and HR) and budding yeast Lif1 (the yeast orthologue of mammalian XRCC4 that is involved in NHEJ). Both Ctp1 and Lif1 have been shown to interact with the N-terminal region of yeast NBS1 (Lloyd et al. 2009; Matsuzaki et al. 2008; Williams et al. 2009). MDC1 orthologues have so far not been detected in yeast. It is currently also not known if human NBS1 is capable of interacting with the human orthologues of yeast Ctp1 and Lif1 (CtIP and XRCC4, respectively) in a CK2-dependent manner.

Doubly phosphorylated pSDpTD peptides interact with both FHA and BRCT domains of human NBS1 since only mutations in both domains effectively abolished the interaction (Lloyd et al. 2009). Mutation of key residues in the FHA and the tandem BRCT domain similarly reduce the interaction between CK2-phosphorylated MDC1 protein and the MRN complex, thus indicating the requirement of an intact domain structure at the N-terminus of NBS1 for optimal binding (Chapman and Jackson 2008; Lloyd et al. 2009). Moreover, alterations in either FHA or BRCT domains impair relocalization of the MRN complex to IRIF (Cerosaletti and Concannon 2003; Spycher et al. 2008; Wu et al. 2008; Xu et al. 2008). These data point to a dual phospho-dependent interaction mode between MDC1 and the FHA/BRCT region of NBS1.

Functionally, the NBS1 FHA/BRCT region is required for the activation of the intra-S-phase and the G2/M DNA damage checkpoints and is likely also participating in DSB repair. However, the molecular mechanisms underlying these functional implications remain largely elusive. Defective checkpoint activation has been observed in NBS1 FHA and BRCT phospho-binding mutants (Difilippantonio et al. 2007; Wu et al. 2008; Zhao et al. 2002), strongly suggesting that the interaction with MDC1 may be required for ensuing an efficient DNA damage checkpoint response. However, it is not yet clear how exactly MDC1-NBS1 complex formation is implicated in DNA damage signaling. One possibility is that accumulation and retention of the MRN complex in chromatin regions flanking DSBs may help to amplify the checkpoint response in the presence of low numbers of DNA breaks (Spycher et al. 2008; Stucki and Jackson 2006). In addition, MDC1-dependent chromatin retention of NBS1 may also be necessary to efficiently recruit the ATM kinase to the damaged chromatin compartments (Falck et al. 2005), even though this issue is currently still a matter of controversy (see also below).

\section{The N-terminal FHA domain: an enigma at the end}

Perhaps with the exception of the PST repeat region, the Nterminal FHA domain of MDC1 is still the most enigmatic element of this large and versatile mediator protein. This is surprising given that FHA domains are well-characterized phospho-specific interaction modules that occur rather frequently in DDR proteins. It has also been clear for a while that the N-terminal FHA domain is important for MDC1's functions in the DDR: deletion of this domain leads to multiple DDR defects, including a defective G2/M DNA damage checkpoint, inefficient DSB repair by sister chromatid recombination, and reduced apoptosis in response to IR (Lou et al. 2003, 2006; Xie et al. 2007; Zhang et al. 2005). Even though several interaction partners for the FHA domain have been identified in the past years, none of them seems to quite "fit in."

As a first candidate, phosphorylated CHK2 was described as putative MDC1-FHA interacting factor (Lou et al. 2003). Immunoprecipitation and peptide-binding studies suggested that CHK2, phosphorlyated on Thr68, could form a stable complex with MDC1 in vitro and in cell extracts. Moreover, the MDC1 FHA domain was required for this interaction. In support of this finding, oriented phosphopeptide library screening revealed that the MDC1 FHA domain bound selectively to peptides containing a phosphorylated Thr residue, and furthermore, selected for Gln at the +1 position and Leu/Ile at the +3 position after the phosphorylated Thr (Durocher et al. 2000). These binding preferences closely match the sequence surrounding the Thr68 of CHK2 (pT-Q-E-L), thus corroborating the interpretation that CHK2 phosphorylated on Thr68 is a bona fide binding partner of the MDC1 FHA domain. However, CHK2 and MDC1 do not co-localize in cells that have been treated by DSB-inducing agents. While MDC1 accumulates in IRIF, CHK2 remains dispersed throughout the nucleus (Lukas et al. 2003). This implies that the interaction between MDC1 and phosphorylated CHK2 is very transient in nature (at least in vivo) and, thus, does not compare, for example, to the stable interaction between the phosphorylated MDC1 TQXF cluster and the RNF8 FHA domain, even though this latter interaction is also based on a phosphorylated TQ motif and an FHA domain. Furthermore, it was recently shown that a tight pThr68-dependent head-to-tail dimerization of CHK2 results in effective occlusion of the phospho-epitope and would prevent 
interaction of the phospho-Thr68 motif with the MDC1 FHA domain ( $\mathrm{Li}$ et al. 2008). However, autophosphorylation of CHK2 releases monomeric CHK2 (Ahn and Prives 2002; Li et al. 2008), which would expose the phosphoThr68 motif and, potentially, allow binding to the MDC1 FHA domain. This mechanism could cause a very rapid switching of the phosphorylated CHK2 between MDC1bound state and MDC1-free dimeric state and may account for the observation that, in response to DNA damage, CHK2 remains dispersed throughout the nucleus and does not accumulate in IRIF (Lukas et al. 2003).

MDC1 was also reported to interact with the ATM kinase through its N-terminal FHA domain (Lou et al. 2006). Immunoprecipitation experiments showed that ATM and MDC1 exist in a complex (Stewart et al. 2003). Moreover, ATM seems to weakly associate with the isolated FHA domain of MDC1 in vitro (Lou et al. 2006). Immunofluorescence microscopy revealed that, in the absence of MDC1 (or in the presence of an MDC1 version lacking the N-terminal FHA domain), antibodies raised against the autophosphorylated ATM kinase could not anymore detect phospho-ATM IRIF accumulation, indicating that MDC1 may recruit phosphorylated ATM into damaged chromatin compartments via direct interaction with its FHA domain. However, it should be pointed out that a correct interpretation of these data is difficult because it is currently very difficult to control the specificity of phospho-specific ATM antibodies in indirect immunofluorescence. Phospho-specific antibodies raised against pTQ/ pSQ peptides are notoriously promiscuous and usually cross-react with many PIKK target proteins (Matsuoka et al. 2007). It is therefore likely that phospho-specific ATM antibodies are no exception and may be recognizing other proteins besides ATM in immunofluorescence. Thus, it is not yet clear if ATM IRIF formation is really dependent on the MDC1 FHA domain. To complicate matters further, it has also been suggested that ATM accumulation at sites of DSBs is mediated by the C-terminal region of NBS1. ATM IRIF formation was defective in cells expressing a NBS1 version lacking a C-terminal ATM-interacting peptide (Falck et al. 2005). Since NBS1 IRIF formation is also dependent on MDC1, it is possible that ATM accumulation is not mediated by direct interaction with MDC1, but indirectly, through its association with the NBS1 Cterminus. To resolve this matter in an unbiased manner, ATM IRIF formation needs to be analyzed with antibodies raised against the non-phosphorylated protein, whose specificity can be properly controlled in ATM-deficient cells. Alternatively, GFP-tagged recombinant ATM may be used to test if IRIF accumulation is dependent upon the SDT region of MDC1 (that mediates the accumulation of the MRN complex, see above), or upon the FHA domain of MDC1 that may directly interact with ATM.
The MDC1 FHA domain has also been implicated in DSB repair via sister chromatid recombination (Xie et al. 2007; Zhang et al. 2005). It was proposed that this is brought about through a specific interaction between the FHA domain of MDC1 and the recombinase RAD51 (Zhang et al. 2005). However, this is difficult to comprehend because, at early time points after irradiation, RAD51 foci and MDC1 IRIF do not co-localize (Goldberg et al. 2003). Moreover, RAD51 and MDC1 do not occupy the same compartments at sites of DSBs: while RAD51 binds to single-stranded DNA stretches that are the result of DSB resection in S and G2 phases of the cell cycle, MDC1 accumulates in the chromatin compartment flanking sites of DSBs, independently of the cell cycle state (Bekker-Jensen et al. 2006). Thus, whether and how interaction between the MDC1 FHA domain and RAD51 contributes to DSB repair by HR remains elusive.

\section{Concluding remarks}

In the past few years, MDC1 has emerged as a prototype mediator, acting mainly as a "molecular matchmaker" in the mammalian response to DNA damage. The entire protein appears to be composed of regions and domains that are involved in protein-protein interactions; most of them occur as phosphorylation-specific interactions.

Recent key findings have also significantly contributed to our understanding as to how MDC1 functions in the DDR. The best-characterized functional role of this protein is that it acts as a bridging element to dynamically tether various DDR factors to damaged chromatin regions that contain phosphorylated H2AX molecules. Mechanistically, this function is based upon a very specific interaction between the MDC1 C-terminal tandem BRCT domain and the phosphorylated H2AX C-terminus. Moreover, several domains and regions within the central and $\mathrm{N}$-terminal sections of MDC1 feature repeated sequence motifs that are shaped by post-translational modifications, mostly phosphorylations. These regions are then recognized by proteins that contain domains capable of specifically binding to the modified amino acid stretches such as FHA and BRCT tandem domains. These factors subsequently accumulate at sites of DSBs and, thus, the $\gamma \mathrm{H} 2 \mathrm{AX}$ chromatin domain is efficiently established.

While the mechanism of accumulation of DDR factors in damaged chromatin and the role of MDC1 in this process is now relatively well understood, it is much more difficult to implicate these processes to the physiological roles of MDC1. While we do know that MDC1 is important for the activation of the intra-S phase and G2/M DNA damage checkpoint and for DSB repair, it is not clear yet how exactly these physiological roles are connected to MDC1's 
major task to control the assembly of the $\gamma \mathrm{H} 2 \mathrm{AX}$ chromatin domain. This is especially true for checkpoint activation. To make this point clear, we touch on just one example: As outlined above, MDC1 is implicated in the G2/M DNA damage checkpoint. Theoretically, this function could be mediated by 53BP1 or BRCA1 accumulation because both 53BP1 and BRCA1 are implicated in the G2/ $M$ checkpoint response and mutation of the TQXF cluster of MDC1 that is essential for 53BP1 and BRCA1 accumulation triggers a $\mathrm{G} 2 / \mathrm{M}$ checkpoint defect. However, deletion of the MDC1 FHA domain also triggers a $\mathrm{G} 2 / \mathrm{M}$ checkpoint defect, which might be related to the ability of the FHA domain to interact with ATM and CHK2, factors that are also critical to induce a proper $\mathrm{G} 2 / \mathrm{M}$ checkpoint response. To complicate matters further, MDC1 also mediates the accumulation of the MRN complex via direct interaction of its phosphorylated SDT repeats with the FHA/BRCT region of NBS1. Mutation in the FHA domain of NBS1 also results in a G2/M checkpoint defect, indicating that MDC1-mediated accumulation of NBS1 in damaged chromatin regions may be required for proper G2/ $M$ checkpoint activation. The relative contribution of these protein-protein interactions is not yet clear and no firm mechanistic explanation is yet available as to how MDC1mediated accumulation of DDR factors at sites of DSBs brings about an efficient $\mathrm{G} 2 / \mathrm{M}$ checkpoint response.

Thus, while MDC1 has clearly emerged as the major organizer of the assembly and maintenance of the $\gamma \mathrm{H} 2 \mathrm{AX}$ chromatin domain, we still need to learn a lot more about how these events translate into a timely and efficient DDR that protects cells from the deleterious and dangerous effects of DNA damage and chromosomal instability.

Acknowledgements We thank Flurina Hari for critical reading of the manuscript. Research in the Stucki laboratory is supported by the Swiss National Foundation and by the Kanton of Zürich.

Conflict of interest The authors declare that they have no conflicts of interest.

\section{References}

Ahn J, Prives C (2002) Checkpoint kinase 2 (Chk2) monomers or dimers phosphorylate $\mathrm{Cdc} 25 \mathrm{C}$ after DNA damage regardless of threonine 68 phosphorylation. J Biol Chem 277(50):4841848426

Bartkova J, Hořejsí Z, Sehested M, Nesland JM, Rajpert-De Meyts E, Skakkebæk NE, Stucki M, Jackson S, Lukas J, Bartek J (2007) DNA damage response mediators MDC1 and 53BP1: constitutive activation and aberrant loss in breast and lung cancer, but not in testicular germ cell tumours. Oncogene 26(53):7414-7422

Bekker-Jensen S, Lukas C, Kitagawa R, Melander F, Kastan MB, Bartek J, Lukas J (2006) Spatial organization of the mammalian genome surveillance machinery in response to DNA strand breaks. J Cell Biol 173(2):195-206
Botuyan MV, Lee J, Ward IM, Kim JE, Thompson JR, Chen J, Mer G (2006) Structural basis for the methylation state-specific recognition of histone H4-K20 by 53BP1 and $\mathrm{Crb} 2$ in DNA repair. Cell 127(7):1361-1373

Cerosaletti KM, Concannon P (2003) Nibrin forkhead-associated domain and breast cancer $\mathrm{C}$-terminal domain are both required for nuclear focus formation and phosphorylation. J Biol Chem 278(24):21944-21951

Chapman J, Jackson S (2008) Phospho-dependent interactions between NBS1 and MDC1 mediate chromatin retention of the MRN complex at sites of DNA damage. EMBO Rep 9(8):795801

Cook P, Ju B, Telese F, Wang X, Glass C, Rosenfeld M (2009) Tyrosine dephosphorylation of H2AX modulates apoptosis and survival decisions. Nature 458(7238):591-596

Coster G, Hayouka Z, Argaman L, Strauss C, Friedler A, Brandeis M, Goldberg M (2007) The DNA damage response mediator MDC1 directly interacts with the anaphase-promoting complex/cyclosome. J Biol Chem 282(44):32053-32064

de Jager M, van Noort J, van Gent DC, Dekker C, Kanaar R, Wyman C (2001) Human Rad50/Mre11 is a flexible complex that can tether DNA ends. Mol Cell 8(5):1129-1135

Difilippantonio S, Celeste A, Kruhlak M, Lee Y, Difilippantonio MJ, Feigenbaum L, Jackson SP, McKinnon PJ, Nussenzweig A (2007) Distinct domains in Nbs1 regulate irradiation-induced checkpoints and apoptosis. J Exp Med 204(5):1003-1011

Dimitrova N, de Lange T (2006) MDC1 accelerates nonhomologous end-joining of dysfunctional telomeres. Genes \& Development 20(23):3238-3243

Dimitrova N, de Lange T (2008) 53BP1 promotes non-homologous end joining of telomeres by increasing chromatin mobility. Nature 456(7221):524-528

Doil C, Mailand N, Bekker-Jensen S, Menard P, Larsen D, Pepperkok R, Ellenberg J, Panier S, Durocher D, Bartek J (2009) RNF168 binds and amplifies ubiquitin conjugates on damaged chromosomes to allow accumulation of repair proteins. Cell 136(3):435446

Dronamraju R, Mason JM (2009) Recognition of double strand breaks by a mutator protein (MU2) in Drosophila melanogaster. PLoS Genet 5(5):e1000473

Durocher D, Jackson S (2002) The FHA domain. FEBS Lett 513 (1):58-66

Durocher D, Taylor IA, Sarbassova D, Haire LF, Westcott SL, Jackson SP, Smerdon SJ, Yaffe MB (2000) The molecular basis of FHA domain: phosphopeptide binding specificity and implications for phospho-dependent signaling mechanisms. Mol Cell 6(5):11691182

Falck J, Coates J, Jackson S (2005) Conserved modes of recruitment of ATM, ATR and DNA-PKcs to sites of DNA damage. Nature 434(7033):605-611

Goldberg M, Stucki M, Falck J, D'amours D, Rahman D, Pappin D, Bartek J, Jackson S (2003) MDC1 is required for the intra-Sphase DNA damage checkpoint. Nature 421(6926):952-956

Gottlieb TM, Jackson SP (1993) The DNA-dependent protein kinase: requirement for DNA ends and association with $\mathrm{Ku}$ antigen. Cell 72(1):131-142

Huen M, Grant R, Manke I, Minn K, Yu X, Yaffe M, Chen J (2007) RNF8 transduces the DNA-damage signal via histone ubiquitylation and checkpoint protein assembly. Cell 131(5):901-914

Huyen Y, Zgheib O, Ditullio RA, Gorgoulis VG, Zacharatos P, Petty TJ, Sheston EA, Mellert HS, Stavridi ES, Halazonetis TD (2004) Methylated lysine 79 of histone H3 targets 53BP1 to DNA double-strand breaks. Nature 432(7015):406-411

Kim H, Chen J, Yu X (2007) Ubiquitin-binding protein RAP80 mediates BRCA1-dependent DNA damage response. Science 316(5828):1202-1205 
Kolas N, Chapman J, Nakada S, Ylanko J, Chahwan R, Sweeney F, Panier S, Mendez M, Wildenhain J, Thomson T, Pelletier L, Jackson S, Durocher D (2007) Orchestration of the DNA-damage response by the RNF8 ubiquitin ligase. Science 318(5856):1637-1640

Lee MS, Edwards RA, Thede GL, Glover J (2005) Structure of the BRCT repeat domain of MDC1 and its specificity for the free $\mathrm{COOH}$-terminal end of the gamma-H2AX histone tail. J Biol Chem 280(37):32053-32056

Li J, Taylor IA, Lloyd J, Clapperton JA, Howell S, MacMillan D, Smerdon SJ (2008) Chk2 oligomerization studied by phosphopeptide ligation: implications for regulation and phosphodependent interactions. J Biol Chem 283(51):36019-36030

Lloyd J, Chapman J, Clapperton JA, Haire LF, Hartsuiker E, Li J, Carr AM, Jackson S, Smerdon SJ (2009) A supramodular FHA/ BRCT-repeat architecture mediates Nbs1 adaptor function in response to DNA damage. Cell 139(1):100-111

Lou Z, Minter-Dykhouse K, Wu X, Chen J (2003) MDC1 is coupled to activated CHK2 in mammalian DNA damage response pathways. Nature 421(6926):957-961

Lou Z, Chen BP, Asaithamby A, Minter-Dykhouse K, Chen DJ, Chen J (2004) MDC1 regulates DNA-PK autophosphorylation in response to DNA damage. J Biol Chem 279(45):46359-46362

Lou Z, Minter-Dykhouse K, Franco S, Gostissa M, Rivera MA, Celeste A, Manis JP, van Deursen J, Nussenzweig A, Paull TT, Alt FW, Chen J (2006) MDC1 maintains genomic stability by participating in the amplification of ATM-dependent DNA damage signals. Mol Cell 21(2):187-200

Lukas C, Falck J, Bartkova J, Bartek J, Lukas J (2003) Distinct spatiotemporal dynamics of mammalian checkpoint regulators induced by DNA damage. Nat Cell Biol 5(3):255-260

Lukas C, Melander F, Stucki M, Falck J, Bekker-Jensen S, Goldberg M, Lerenthal Y, Jackson S, Bartek J, Lukas J (2004) Mdc1 couples DNA double-strand break recognition by Nbs1 with its H2AXdependent chromatin retention. EMBO J 23(13):2674-2683

Luo K, Yuan J, Chen J, Lou Z (2009) Topoisomerase IIalpha controls the decatenation checkpoint. Nat Cell Biol 11(2):204-210

Mailand N, Bekkerjensen S, Faustrup H, Melander F, Bartek J, Lukas C, Lukas J (2007) RNF8 ubiquitylates histones at DNA doublestrand breaks and promotes assembly of repair proteins. Cell 131 (5):887-900

Matsuoka S, Ballif BA, Smogorzewska A, McDonald ER, Hurov KE, Luo J, Bakalarski CE, Zhao Z, Solimini N, Lerenthal Y, Shiloh Y, Gygi SP, Elledge SJ (2007) ATM and ATR substrate analysis reveals extensive protein networks responsive to DNA damage. Science 316(5828):1160-1166

Matsuzaki K, Shinohara A, Shinohara M (2008) Forkhead-associated domain of yeast Xrs2, a homolog of human Nbs1, promotes nonhomologous end joining through interaction with a ligase IV partner protein, Lif1. Genetics 179(1):213-225

Meier A, Fiegler H, Muñoz P, Ellis P, Rigler D, Langford C, Blasco M, Carter N, Jackson S (2007) Spreading of mammalian DNAdamage response factors studied by ChIP-chip at damaged telomeres. EMBO J 26(11):2707-2718

Melander F, Bekker-Jensen S, Falck J, Bartek J, Mailand N, Lukas J (2008) Phosphorylation of SDT repeats in the MDC1 N terminus triggers retention of NBS1 at the DNA damage-modified chromatin. J Cell Biol 181(2):213-226

Minter-Dykhouse K, Ward I, Huen SY, Chen J, Lou Z (2008) Distinct versus overlapping functions of $\mathrm{MDC} 1$ and $53 \mathrm{BP} 1$ in DNA damage response and tumorigenesis. J Cell Biol 181(5):727-735

Mohammad D, Yaffe MB (2009) 14-3-3 proteins, FHA domains and BRCT domains in the DNA damage response. DNA Repair 8 (9):1009-1017

Nakada S, Chen GI, Gingras AC, Durocher D (2008) PP4 is a gamma $\mathrm{H} 2 \mathrm{AX}$ phosphatase required for recovery from the DNA damage checkpoint. EMBO Rep 9(10):1019-1026
Panier S, Durocher D (2009) Regulatory ubiquitylation in response to DNA double-strand breaks. DNA Repair 8(4):436-443

Rodriguez M, Yu X, Chen J, Songyang Z (2003) Phosphopeptide binding specificities of BRCA1 COOH-terminal (BRCT) domains. J Biol Chem 278(52):52914-52918

Rogakou EP, Boon C, Redon C, Bonner WM (1999) Megabase chromatin domains involved in DNA double-strand breaks in vivo. J Cell Biol 146(5):905-916

Savic V, Yin B, Maas N, Bredemeyer A, Carpenter A, Helmink B, Yang-Iott K, Sleckman B, Bassing C (2009) Formation of dynamic $\gamma$-H2AX domains along broken DNA strands is distinctly regulated by ATM and MDC1 and dependent upon H2AX densities in chromatin. Mol Cell 34(3):298-310

Shang YL, Bodero AJ, Chen PL (2003) NFBD1, a novel nuclear protein with signature motifs of FHA and BRCT, and an internal 41-amino acid repeat sequence, is an early participant in DNA damage response. J Biol Chem 278(8):6323-6329

Sobhian B, Shao G, Lilli DR, Culhane AC, Moreau LA, Xia B, Livingston DM, Greenberg RA (2007) RAP80 targets BRCA1 to specific ubiquitin structures at DNA damage sites. Science 316 (5828):1198-1202

Spycher C, Miller ES, Townsend K, Pavic L, Morrice NA, Janscak P, Stewart GS, Stucki M (2008) Constitutive phosphorylation of MDC1 physically links the MRE11-RAD50-NBS1 complex to damaged chromatin. J Cell Biol 181(2):227-240

Stewart GS (2009) Solving the RIDDLE of 53BP1 recruitment to sites of damage. Cell Cycle 8(10):1532-1538

Stewart GS, Wang B, Bignell CR, Taylor AM, Elledge SJ (2003) MDC1 is a mediator of the mammalian DNA damage checkpoint. Nature 421(6926):961-966

Stewart GS, Stankovic T, Byrd PJ, Wechsler T, Miller ES, Huissoon A, Drayson MT, West S, Elledge SJ, Taylor AM (2007) RIDDLE immunodeficiency syndrome is linked to defects in 53BP1mediated DNA damage signaling. Proc Natl Acad Sci USA 104 (43):16910-16915

Stewart G, Panier S, Townsend K, Al-Hakim A, Kolas N, Miller E, Nakada S, Ylanko J, Olivarius S, Mendez M (2009) The RIDDLE syndrome protein mediates a ubiquitin-dependent signaling cascade at sites of DNA damage. Cell 136(3):420 434

Stucki M (2009) Histone H2A.X Tyr142 phosphorylation: a novel sWItCH for apoptosis? DNA Repair 8(7):873-876

Stucki M, Jackson S (2004) MDC1/NFBD1: a key regulator of the DNA damage response in higher eukaryotes. DNA Repair 3(8-9):953-957

Stucki M, Jackson S (2006) $\gamma \mathrm{H} 2 \mathrm{AX}$ and MDC1: anchoring the DNAdamage-response machinery to broken chromosomes. DNA Repair 5(5):534-543

Stucki M, Clapperton J, Mohammad D, Yaffe M, Smerdon S, Jackson S (2005) MDC1 directly binds phosphorylated histone H2AX to regulate cellular responses to DNA double-strand breaks. Cell 123(7):1213-1226

Townsend K, Dyson H, Blackford AN, Miller ES, Chapman JR, Sedgwick GG, Barone G, Turnell AS, Stewart GS (2009) Mediator of DNA damage checkpoint 1 (MDC1) regulates mitotic progression. J Biol Chem 284(49):33939-33948

Van Attikum H, Gasser S (2009) Crosstalk between histone modifications during the DNA damage response. Trends Cell Biol 19 (5):207-217

Wang B, Matsuoka S, Ballif BA, Zhang D, Smogorzewska A, Gygi SP, Elledge SJ (2007) Abraxas and RAP80 form a BRCA1 protein complex required for the DNA damage response. Science 316(5828):1194-1198

Williams RS, Williams JS, Tainer JA (2007) Mre11-Rad50-Nbs1 is a keystone complex connecting DNA repair machinery, doublestrand break signaling, and the chromatin template. Biochem Cell Biol 85(4):509-520 
Williams RS, Dodson GE, Limbo O, Yamada Y, Williams JS, Guenther G, Classen S, Glover J, Iwasaki H, Russell P, Tainer JA (2009) Nbs1 flexibly tethers Ctp1 and Mre11-Rad50 to coordinate DNA double-strand break processing and repair. Cell 139(1):87-99

Wu L, Luo K, Lou Z, Chen J (2008) MDC1 regulates intra-S-phase checkpoint by targeting NBS1 to DNA double-strand breaks. Proc Natl Acad Sci USA 105(32):11200-11205

Xiao A, Li H, Shechter D, Ahn S, Fabrizio L, Erdjument-Bromage H, Ishibe-Murakami S, Wang B, Tempst P, Hofmann K, Patel D, Elledge SJ, Allis C (2009) WSTF regulates the H2A.X DNA damage response via a novel tyrosine kinase activity. Nature 457 (7225):57-62

Xie A, Hartlerode A, Stucki M, Odate S, Puget N, Kwok A, Nagaraju G, Yan C, Alt FW, Chen J, Jackson S, Scully R (2007) Distinct roles of chromatin-associated proteins $\mathrm{MDC} 1$ and 53BP1 in mammalian double-strand break repair. Mol Cell 28(6):10451057

Xu C, Wu L, Cui G, Botuyan M, Chen J, Mer G (2008) Structure of a second BRCT domain identified in the nijmegen breakage syndrome protein Nbs1 and its function in an MDC1-dependent localization of Nbs1 to DNA damage sites. J Mol Biol 381 (2):361-372

Zhang J, Ma Z, Treszezamsky A, Powell SN (2005) MDC1 interacts with Rad51 and facilitates homologous recombination. Nat Struct Mol Biol 12(10):902-909

Zhao S, Renthal W, Lee EY (2002) Functional analysis of FHA and BRCT domains of NBS1 in chromatin association and DNA damage responses. Nucleic Acids Res 30(22):4815-4822

Zhou BB, Elledge SJ (2000) The DNA damage response: putting checkpoints in perspective. Nature 408(6811):433-439 